\title{
Segmentation and Classification of Brain Tumour Images
}

\author{
Shreeja. M, Mrs. Padmanayana
}

Computer Science and Engineering, Srinivas Institute of Technology Valachil, Mangalure, Karnaatka, India

\section{Article Info}

Volume 7, Issue 4

Page Number : 297-302

\section{Publication Issue :}

July-August-2021

\section{Article History}

Accepted : 10 July 2021

Published : 17 July 2021

\section{ABSTRACT}

A tumor in the brain is an uncontrolled and abnormal cell proliferation in the brain and are categorized into four levels. Each tumor grade has its own set of contrast variations, that are captured using the MRI technology. Different computational models are used to accurately segment these cancers and to classify it. In this research, we present a method for segmenting brain tumors that uses a model based on deep learning called U-Net, and also classification of the segmented images is done using Random-Forest classifier. The proposed method was tested on the Brain Tumor Image Segmentation (BRATS) 2015 dataset and showed to be effective. With the best overall accuracy of 77 percent, the proposed network structure achieves a remarkable performance.

Keywords: Brain tumor, U-Net, Random Forest

\section{INTRODUCTION}

A brain tumor is a heinous disease that causes death in most of the cases due to lack of proper diagnosis and treatment. It happens when aberrant cells in the brain form. Cancerous (malignant) tumors and benign (non-cancerous) tumors are the two basic forms of tumors. Brain tumors that begin in the cerebrum are called malignant tumor and tumors that begin in other part of the body and then spread to the brain are called benign tumors.

Necrosis, edoema, non-enhancing, and enhancing tumours are the four categories of brain tumours, and tumours are intimated as high grade glioma (HGG) or low grade glioma (LGG)[1]. To find these malignancies, The soft tissues are imaged using Magnetic Resonance Imaging (MRI), which provides strong contrast. For the detection of brain cancers, different MRI modalities such as T1, T1c, T2, and Tf images are combined. Each modality produces various contrast changes for different types of brain tissues.

MRI imaging has traditionally been used by researchers. In computational models, modalities are combined for the segmentation of brain tumours [2][4]. These studies miss one of the most crucial aspects of using a single modality for particular tumoral locations, which could improve the model's performance. The basis for this decision is that each modality provides distinct contrast to various parts of the brain. Some contrast differences that aren't caught in one modality may exist in another. In this paper, BRATS 2015 dataset is used to test our technique.

The authors of [10] devised a fairly decent technique based on the U-Net architecture, a 2D convolutional 
based segmentation model. In their procedure, they used a number of data expansion techniques, which resulted in high dice counts. For various grades of brain tumours, this approach produced the best segmentation results. After the segmentation, the images are classified using Random-Forest classifier.

\section{METHODS AND MATERIAL}

Segmentation and classification of brain image is necessary as high accuracy is needed. The image is segmented first and then features are extracted and classification of the images are done using random forest algorithm.

We offer a one-of-a-kind system that evaluates distinct modalities for different grades of malignancies using a changed version of the U-Net construction. The suggested technique is depicted in Figure 1 as a high-level overview. There are four important steps in the suggested technique: 1) Tumour Segmentation, 2) Trained Weights, 3) U-Net Planning, and 4) MRI Pre-processing.

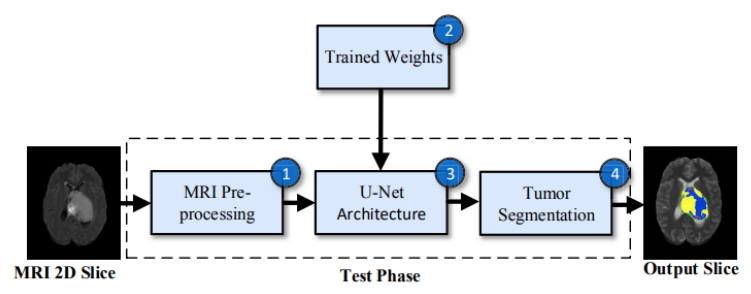

Figure 1: Steps involved in the segmentation process.

\section{A. MRI Pre-processing}

As input, this step uses an axial MRI piece obtained from a 3D MRI picture. The raw MRI pieces show a wide range of values, different levels of detail (which differ from slice to slice), and low contrast values in a large dynamic scope. The slices must be preprocessed to remove undesired artefacts and ensure that the incoming data is homogeneous. The following three steps are used to pre-process input MRI slices.

\section{Extraction of detailed slices}

The slices were taken from the whole BRATS2015 HGG dataset, which contains 220 3D photos of patients. The image of each subject has $1502 \mathrm{D}$ slices. Only the most information-dense slices of the brain with a significant percentage of the brain were chosen.

2.Variance stretching

To improve the low contrast in high dynamic range values, contrast stretching is claimed to all retrieved MRI slabs. The contrast of each slice is increased to the maximum range by this operation.

\section{Standardization}

Standardization is used to plate all pixel values between 0 and 1 . The impact of the aspects was so balanced. We collect feature maps from each raw pixel of a slice during the convolutions of U-Net building, and by normalising each image, we get the influence of each pixel in a given range. This allows the classifier to recognise the differences between each photo right away.

\section{B. Weights that have been trained}

The pre-processed pieces from the previous phase are used to create the trained weights vector. The proposed U-Net building is trained using the preprocessed slices and the ground truth associated with them. The training weights are then employed in the model to detect and segment four different forms of brain tumours, as shown in Figure 1.

\section{U-Net planning}

To detect if the pieces have tumours and segment them, this phase uses the pre-processed slices and trained weight vector from the preceding methods. The HGG and LGG brain tumours are segmented using this U-Net architecture [6]. A down and up sampling mechanism is used in the suggested design (as illustrated in Figure 2). The down sampling 
procedure is broken into five blocks, each of which performs comparable tasks. To reduce spatial resolution and increase feature map size, each slice of an input tensor goes through two layers of convolutions and one layer of max pooling operations at each of the five blocks. This procedure continues until the sixth block emerges, at which point upsampling takes over for the remaining five slabs. Up sampling runs each block through a transpose convolution, which reduces the size of the feature map as the spatial resolution improves. Additionally, two levels of convolutions with varied filter sizes are conducted by upsampling till the output layer is reached. Finally, a 1x1 kernel is used, with filters equal to the number of classes, in this case five (one normal class and four tumour classes).

Different parameters are utilised in each phase of the proposed architecture. The following sections go over how to choose parameters as well as other pertinent information:

1) Activation function: The Rectifier linear unit (ReLU) [7] activation function is used in our copy.

2) Down Sampling: Our model divides down sampling into five blocks, each of which executes similar repeating procedures. For down sampling, a pooling procedure is utilised to reduce computational load. Max pooling operation is used for downsampling.

3) Up sampling: In our concept, up sampling is separated into five blocks, each of which performs an augmentation operation to improve resolution.

4) Regularization: To generalise the classifier, we use regularisation procedures, which eliminate a certain amount of signal to stop model overfitting. In our model, batch normalisation [9] is used for regularisation.

5) Minimization function: A loss must be reduced over the training iterations throughout the training phase. To obtain a rapid optimization of the error surface, we employ adam optimizer [9]. The model generates a segmented map at the output coat by down and up sampling the input tensor many times. This method is performed to reduce the error between pixels in the ground truth image and the produced output image. Once the model's error has been converged for all classes, the training weights are saved.

\section{D. malignancy segmentation}

The U-Net design creates a segmented map of tumour regions, with each pixel representing one of five different forms of tumour. Each pixel's price ranges from zero to four, with zero representing regular elegance, one representing necrosis, two representing edoema, three representing non-improving, and four representing improving grade tumour. The tumours connected with each aspect are pseudo-colored to visually see the subdivided regions according on the prior information.

\section{E. Feature Extraction}

We need to extract features from the images because we need to make a binary classification of them using a classifier that needs to be trained on these features. We choose to extract GLCM(texture-based features). GLCM features: Once the segmented images are obtained from the $\mathrm{u}$-net architecture, GLCM features are extracted and stored. Analysis of Texture The Gray-Level Co-Occurrence Matrix is a statistical texture analysis method that takes into account the spatial interaction of pixels.

\section{F. Classification}

For classification, we used Random Forest classifier[14]. The classifier was trained to discriminate between benign (LGG) and malignant (MLG) tumours using GLCM characteristics taken from segmented images (HGG).Figure2 shows the flowchart of the proposed method. 
When the training set for the current tree is drawn using sampling with replacement, about one-third of the cases are left out of the sample. This oob (out-ofbag) data is utilised to derive a running unbiased estimate of the classification error as trees are added to the forest. It's also used to measure the relevance of variables. All of the data is run down the tree once each tree is formed, and proximities for each pair of cases are computed. The distance between two cases is increased by one when they share a terminal node. At the end of the run, the proximities are normalised by dividing by the number of trees. Proximities are used to fill in blanks, find outliers, and construct informative low-dimensional data representations.

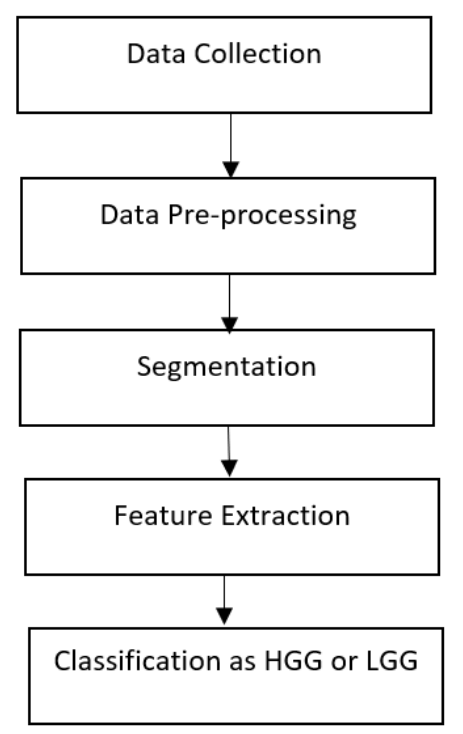

Figure 2: Flow chart of the proposed method

\section{RESULTS AND DISCUSSION}

\section{A. Data Set}

To conduct our investigations, we used the BRATS 2015 dataset [5]. T1, T1c, T2, and Tf MRI imaging modalities, as well as ground truth, are included in the dataset. HGG (High Grade Glioma) and LGG (Low Grade Glioma) patients are separated in this dataset. The HGG has 220 patients, whereas the LGG has 54 patients, each of whom has 155 slices. Each slice has a resolution of 240 by 240 pixels and a bit depth of 16.

\section{B. SetUp}

220 HGG patient photos and 54 LGG patient photos are used for training. Before training our model, images are separated into discrete slices and preprocessed. For testing, brain images of 110 patients are used. There are four different MRI images for each patient. The four different images are T1,T2, T1c and FLAIR.

\section{Discussion}

We decided to use only the training dataset for both training and testing because our testing dataset lacks ground truth labels. Because there are only 54 photographs in LGG and 220 images in HGG, we chose to take into account all 54 images in LGG and only 54 out of 220 images in HGG. We now have a total of 54 LGG and 54 HGG photos. We used 50 HGG and 50 LGG photos to train our model. Testing is done with the remaining 4 HGG and 4 LGG. The outcomes are depicted in the diagram below.

We derived measures like Accuracy, Precision, Recall, and F1-Score to evaluate our model. Below are the results for six separate training datasets and their corresponding testing datasets. The accuracy of the model is nearly $77 \%$.

The most straightforward performance metric is accuracy, which is just the proportion of correctly predicted observations to all observations.

Precision is defined as the ratio of successfully anticipated positive observations to total expected positive observations.

The ratio of accurately predicted positive observations to all observations in the actual class is known as recall. 
The weighted average of Precision and Recall is the F1 Score. As a result, this score considers both false positives and false negatives.

Accuracy $=\mathrm{TP}+\mathrm{TN} / \mathrm{TP}+\mathrm{FP}+\mathrm{FN}+\mathrm{TN}$

Precision $=\mathrm{TP} / \mathrm{TP}+\mathrm{FP}$

Recall $=\mathrm{TP} / \mathrm{TP}+\mathrm{FN}$

F1 Score $=2^{*}\left(\right.$ Recall ${ }^{*}$ Precision $) /($ Recall + Precision $)$

True Positive refers to the number of positive expected events that really occur (TP). The number of expected negative cases that are also genuine negative is referred to as True Negative (TN). False Negative (FN) is the number of projected negative situations that turn out to be positive, also known as (type two) error. False Positive (FP) is the number of projected positive cases that turn out to be negative, also known as (type one) error.

\section{IV.CONCLUSION}

In this work, we have presented a U-net segmentation architecture for the segmentation of brain images. Then segmented images are classified using Random-Forest classifier as Benign or Malignant. It significantly reduces the amount of time that a doctor takes to identify and classify the tumour. It also to some extent reduces the error made while segmenting and classifying tumour. The highest accuracy of 77.77 percent was reached by our proposed approach.

\section{REFERENCES}

[1] S. Bauer, R. Wiest, L.-P. Nolte, and M. Reyes, "A survey of mri-based medical image analysis for brain tumor studies," Physics in medicine and biology, vol. 58, no. 13, pp. Pages 97-103, 2013

[2] M. Havaei, A. Davy, D. Warde-Farley, A. Biard, A. C. Courville, Y. Bengio, C. Pal, P. Jodoin, and $\mathrm{H}$. Larochelle, "Brain tumor segmentation with deep neural networks," CoRR, vol. abs/1505.03540, 2015. [Online]. Available: http://arxiv.org/abs/1505.03540

[3] M. Havaei, A. Davy, D. Warde-Farley, A. Biard, A. Courville, Y. Bengio, C. Pal, P.-M. Jodoin, and H. Larochelle, "Brain tumor segmentation with deep neural networks," Medical image analysis, vol. 35, pp. 18-31, 2017.

[4] B. Erden, N. Gamboa, and S. Wood, "3d convolutional neural network for brain tumor segmentation," 2017.

[5] B. H. Menze, A. Jakab, S. Bauer, J. KalpathyCramer, K. Farahani, J. Kirby, Y. Burren, N. Porz, J. Slotboom, R. Wiest et al., "The multimodal brain tumor image segmentation benchmark (brats)," IEEE transactions on medical imaging, vol. 34, no. 10, pp. 1993-2024, 2015.

[6] O. Ronneberger, P. Fischer, and T. Brox, "Unet: Convolutional networks for biomedical image segmentation," CoRR, vol. abs/1505.04597, 2015. [Online]. Available: http://arxiv.org/abs/1505.04597

[7] K. He, X. Zhang, S. Ren, and J. Sun, "Delving deep into rectifiers: Surpassing human-level performance on imagenet classification," $C o R R$, vol. abs/1502.01852, 2015.[Online].Available:http://arxiv.org/abs/15 02.01852

[8] S. Ioffe and C. Szegedy, "Batch normalization: Accelerating deep network training by reducing internal covariate shift," in Proceedings of the 32Nd International Conference on International Conference on Machine Learning - Volume 37, ser. ICML'15. JMLR.org, 2015, pp. 448-456. [Online]. Available: http://dl.acm.org/citation.cfm?id= 3045118.3045167

[9] D. P. Kingma and J. Ba, "Adam: A method for stochastic optimization," CoRR, vol. abs/1412.6980, 2014.[Online]. 
[10] H. Dong, G. Yang, F. Liu, Y. Mo, and Y. Guo, "Automatic brain tumor detection and segmentation using $\mathrm{u}$-net based fully convolutional networks," CoRR, vol. abs/1705.03820, 2017.

[11] R. Meier, S. Bauer, J. Slotboom, R. Wiest, and M. Reyes, "Appearanceand context-sensitive features for brain tumor segmentation," Proceedings of MICCAI BRATS Challenge, pp. 020-026, 2014.

[12] S. Pereira, A. Pinto, V. Alves, and C. A. Silva, "Brain tumor segmentation using convolutional neural networks in mri images," vol. 35, no. 5. IEEE, 2016, pp. 1240-1251.

[13] L. Shen, "Multimodal brain mri tumor segmentation via convolutional neural networks," 2017.

[14] https://www.stat.berkeley.edu/breiman/Rando mForests/cc home.htmoverview

\section{Cite this article as :}

Shreeja. M, Mrs. Padmanayana, "Segmentation and Classification of Brain Tumour Images", International Journal of Scientific Research in Computer Science, Engineering and Information Technology (IJSRCSEIT), ISSN : 2456-3307, Volume 7 Issue 4, pp. 297-302, July-August 2021. Available at doi : https://doi.org/10.32628/CSEIT217412 Journal URL : https://ijsrcseit.com/CSEIT217412 